\title{
LAS ASOCIACIONES DE AMIGOS DEL CAMINO DE SANTIAGO. ALTRUISMO Y COLABORACIÓN
}

\author{
Xosé M. Santos* \\ Universidad de Santiago de Compostela \\ https://orcid.org/0000-0001-8088-7454
}

\section{RESUMEN}

El papel de las Asociaciones de Amigos del Camino es muy desconocido en la investigación sobre la peregrinación a Santiago. Su función es crucial para entender la recuperación contemporánea del Camino, su difusión internacional y la conservación de muchas de las singularidades de la ruta. En este artículo, a través de encuestas y entrevistas, se analiza su visión actual de la peregrinación así como sus relaciones con las administraciones públicas y la Iglesia. Su labor, en contacto directo con los peregrinos, hace que tengan un buen conocimiento de las necesidades de éstos, desarrollando acciones de apoyo y orientación al caminante. Sin embargo, frecuentemente, no son tenidas en cuenta por las instituciones que gestionan los itinerarios jacobeos. Se concluye afirmando que la importancia de estas asociaciones es fundamental para mantener la dinámica de la ruta y que deben de ser superados algunos obstáculos para mantener el lugar central que ocupan en el mundo jacobeo.

Palabras clave; Asociaciones de Amigos del Camino; peregrinación; voluntariado; colaboración; altruismo.

\section{The Associations of Friends of the Way to Santiago. Altruism and Collaboration}

\section{ABSTRACT}

The role of the Associations of Friends of the Camino de Santiago is virtually unknown in research into the pilgrimage to Santiago. Their function is crucial for understanding the modern-day recovery of the Camino, its international fame and the conservation of many of the unique features along the way. In this paper, based on surveys and interviews, we analyse their current vision of the pilgrimage as well as their relations with public administrations and the Church. Their work, in direct contact with the pilgrims, means that they have a good

Fecha de recepción: 2 de enero de 2020.

Fecha de aceptación: 1 de septiembre de 2020.

*Departamento de Geografía. Universidad de Santiago de Compostela. Plaza de la Universidad, 1. 15782 SANTIAGO DE COMPOSTELA (España).E-mail: xosemanuel.santos@usc.es 
knowledge of their needs, developing actions to support and guide the wayfarer. However, they are often not taken into account by the institutions that manage the Jacobean itineraries. We conclude by stating that the importance of these associations is fundamental for maintaining the dynamics of the route and that some obstacles must be overcome in order to maintain the central place they occupy in the Jacobean world.

Keywords: Associations of Friends of the Camino; pilgrimage; volunteering; collaborations; altruism.

\section{INTRODUCCIÓN}

El Camino de Santiago ha sido un tema recurrente en la investigación turística. Libros, capítulos de libros y artículos en revistas han abordado las motivaciones y el resurgir de este itinerario religioso adaptado a los tiempos post-seculares, su transformación en ruta cultural y su conversión en un producto turístico de éxito que inspira la puesta en valor de otras rutas (Graham y Murray, 1997; Murray y Graham, 1997; Frey, 1998; Lois-González, 2013; Slavin, 2013;Lois-González y Santos, 2015; Magry, 2015; Kim, Kim y King, 2016; Sanchez y Sanchez y Hesp, 2016;). Los enfoques de estos estudios son muy amplios pero tienen algunos puntos en común que le dan cierta coherencia.Uno de ellos es el carácter contemporáneo, aunque enraizado en la historia, de este fenómeno. Es decir, se busca entender y explicar cómo una vía medieval de peregrinación resulta atractiva para los nuevos perfiles de viajeros del siglo XXI.

En relación con las motivaciones están las dificultades para conceptualizar las figuras del peregrino y del turista. En general, se advierte en la literatura académica una tendencia que dibuja unas fronteras líquidas entre ambas. En este sentido, se puede citar a CollinsKreiner (2018) y toda una serie de investigaciones que ponen en duda esa oposición binaria entre turista y peregrino (Collins-Kreiner, 2010; MaClean, 2009; Terzidou, Scarles y Saunders, 2017; Timothy y Olsen, 2006). Terzidou, Scarles y Saunders (2018) confirman la complejidad de las motivaciones del viaje a los lugares sagrados. Por un lado, se refieren a las motivaciones construidas relacionadas con el poder, que son muy obvias en el Camino de Santiago (Pack, 2010; Santos, 2016; Santos y Trillo-Santamaría, 2017). Pero por otro lado, existen otras que son de carácter más subjetivo y personal, vinculadas por ejemplo con momentos importantes de la vida, constituyendo una especie de rito de paso (Terzidou et al., 2018). También Lois-González y Santos (2015), Blom, Nilsson y Santos (2016) o Nilsson y Tesfahuney (2016), han puesto en relación el post-secularismo con la peregrinación a Santiago. De esta manera, el concepto de espiritualidad se integraría con el del nuevo significado de las religiones.

Todavía en el ámbito de las motivaciones y el consiguiente debate entre turista y peregrino, Howard (2012) relaciona los verdaderos peregrinos con el tiempo, con la falta de preocupación por el mismo que permite entrar en el espacio de la liminialidad. También Dunn (2016) o Olsen y Wilkinson (2016) tratan sobre el concepto de verdaderos o falsos peregrinos. Estos dos últimos autores ponen en relación la idea de verdadero o 
falso peregrino con la velocidad. Si bien advierten que esa conexión parece dominar en la literatura occidental, en sus preguntas finales de si "Are fast pilgrims true pilgrims? Is haste the antithesis of authenticity?" (Olsen y Wilkinson, 2016, p. 229) señalan que la respuesta puede no ser categórica considerando la influencia de la modernidad europea en la construción de la idea de peregrinación.

$\mathrm{El}$ excesivo foco que se ha puesto sobre el peregrino, como figura central del fenómeno peregrinatorio, ha llevado a dejar en un segundo plano otros aspectos relevantes. Uno de ellos es el que tiene que ver con la gobernanza más allá de las relaciones de poder protagonizadas en torno al gobierno formal en el que participan instituciones públicas e Iglesia, que son las protagonistas principales (Santos, 2017). Sin embargo, uno de los actores fundamentales para entender la peregrinación a Santiago y toda la etapa previa a su resurgir contemporáneo, que se inicia a principios de la década de 1990, es frecuentemente olvidado. Se trata de una institución, que bajo la denominación genérica de Asociaciones de Amigos del Camino-AAC, ha tenido un papel principal en la segunda mitad del siglo XX en la recuperación y difusión de la cultura jacobea. Actualmente son más de 300 distribuidas por todo el mundo, desarrollando diversas labores, desde la gestión de albergues o la conservación de las rutas hasta la organización de peregrinaciones o la ayuda espiritual al retorno. Tan solo algunas obras, como la de Frey (1998), le han prestado algunas líneas dejando ver su papel relevante en la atención al peregrino y en la puesta en valor del Camino.

La escasa atención que se le ha dado a las AAC ha evitado abordar temas fundamentales para comprender el fenómeno jacobeo no sólo en su perspectiva temporal sino también espacial, motivacional y de relaciones de poder. Nos referimos, por ejemplo, a su contribución en la difusión internacional de la peregrinación, a su labor en la preservación de valores tradicionales, mas que religiosos, o a la realización de tareas propias de otras instituciones, como la gestión de albergues o el mantenimiento físico de las rutas. El objetivo de esta investigación es entender la función que desempeñan las AAC, así como su papel en el resurgir contemporáneo del Camino y sus relaciones de colaboración, no siempre fáciles, con las instituciones responsables de la gestión de la ruta. Nos interesa, además, reflexionar sobre las consecuencias que podría tener un debilitamiento de las AAC, que basan su funcionamiento en el altruismo y el voluntariado, sobre el futuro de la peregrinación a Compostela.

\section{METODOLOGÍA}

Desde el punto de vista metodológico se usaron técnicas cuantitativas y cualitativas de forma complementaria. Como punto central estuvo la cesión por parte de la administración autonómica gallega de una base de datos con todas las AAC existentes con fecha de enero de 2019. En total 347 de 37 países, correspondiendo el $46.1 \%$ de las entradas a España, el $19.3 \%$ a Francia, el $7.78 \%$ a Brasil, el $5.47 \%$ a Alemania y el $3.74 \%$ a Portugal. Esta elevada concentración en muy pocos países refleja parcialmente la importancia de los distinos mercados (los peregrinos españoles fueron en 2018 el $44 \%$ del total, los alemanes el $7.73 \%$ y los portugueses el $4.4 \%$, por ejemplo) aunque con excepciones relevantes. Una de estas son los Estados Unidos, el $4^{\circ}$ origen más voluminoso pero que sólo cuenta con una potente Asociación, tal vez la más grande, organizada en Capítulos. Frente a este caso, o 
al de Canadá, otros países, muy particularmente España, muestran una gran fragmentación, con un número elevado de AAC, hecho que ya llamaba la atención a Frey (1998).

Se hizo un trabajo selectivo para eliminar alguna duplicidad y otros pequeños problemas detectados en la base de datos. A partir de esta primera depuración el número se redujo en 27, prácticamente todas de España. A las asociaciones identificadas se le envió un cuestionario con 17 preguntas específicas que demandaban opiniones e informaciones relativas a la función actual de las AAC, al papel deseado, a su relación con las administraciones públicas y con la Iglesia y otras más vinculadas con la percepción del Camino y de la peregrinación. Por ejemplo, la motivación, principales problemas del Camino o como mejorar la experiencia de los peregrinos. Aunque las preguntas eran cerradas, la caja de observaciones nos permitió recoger muchos matices que sirvieron para enriquecer nuestro análisis. Según Secor (2010), una encuesta permite alcanzar dos propósitos: “(1) to represent in summary form basic characteristics of research subjects; or (2) to present findings that are statistically valid and accurate for a population" (p. 196).

La encuesta fue lanzada en tres idiomas (español, inglés y francés) a través de una plataforma comercial que incluía recordatorios a los destinatarios. El número de respuestas recibido fue bastante reducido, a penas 36. En un segundo intento, se hizo el envío a través de nuestro correo electrónico institucional. Aplicando el muestreo aleatorio simple y considerando que se trata de una población finita $(\mathrm{N}=320)$, se recibieron $75(\mathrm{n}=75)$ cuestionarios, lo que supone un margen de error del 9,92\% para un nivel de confianza aceptable del $95 \%(\mathrm{z}=1,96)$ y bajo el supuesto de máxima indeterminación $(\mathrm{P}=\mathrm{Q}=50 \%)$.

La procedencia de las encuestas fue representativa del universo de las asociaciones, con un mayor volumen de España y Francia, aunque con algunos matices que responden a las características de las mismas. Para el caso de España, el país con más AAC, el porcentaje de respuestas fue algo menor reflejando una situaciación en la que existe una excesiva fragmentación de asociaciones, muchas de las que son de tamaño muy pequeño. Por el contrario, en Estados Unidos y Canadá, son pocas pero más grandes y su representatividad en la encuesta es mayor. En todo caso, lo importante es que se recibieron de todos las regiones, desde Europa del Este (Eslovaquia, Polonia o Rumanía), Europa Occidental (Italia, Bélgica o Suecia), Asia (China, Filipinas) y América Latina (Brasil o Chile). Esta primera fase se complementó con la consulta a algunas páginas web de las AAC que nos permitió resolver algunas dudas y obtener una visión más clara de su labor.

La segunda fase, la de entrevistas, pretendía, desde una perspectiva cualitativa, profundizar en el conocimiento de las $\mathrm{AAC}$, de sus funciones, problemática, expectativas y propuestas., De carácter semi-estructurada, fueron realizadas a personas destacadas del asociativismo jacobeo, presidentes, fundadores o miembros activos de asociaciones representativas de los principales países emisores de peregrinos (Estados Unidos, Italia, Francia, Alemania). Además, se incluyó también a un trabajador cualificado de la administración regional de Galicia con una larga trayectoria de trabajo con el Camino y con las AAC. De igual forma, fue entrevistado un experto que forma parte de la Fraternidad Internacional del Camino de Santiago, que no es exactamente una AAC pero sí muchos de sus miembros, compartiendo con las AAC objetivos y acciones. En total se realizaron 10 entrevistas con una duración que osciló entre 30 y 60 minutos. Se trata de un número 
reducido pero que nos sirvió para profundizar en los resultados de las encuestas y tener una idea mucho más exacta acerca de la labor de las AAC.

Los trabajos finales de una entrevista, la codificación y la interpretación, son especialmente importantes. En relación a la primera se identificaron los principales temas, ideas y conceptos. A partir de los mismos se procedió a su análisis. Para tal fin se condensaron las ideas clave de cada entrevista en relación a los objetivos de esta investigación y como fuente complementaria de las encuestas. De esta manera, las entrevistas nos permitieron obtener importantes matices y profundizar y enriquecer la información obtenida de una forma más cuantitativa a través de los cuestionarios.

\section{LAS ASOCIACIONES DE AMIGOS DEL CAMINO Y SUS FUNCIONES CLAVE EN LA PEREGRINACIÓN JACOBEA}

En 1950 cuando la peregrinación a Santiago estaba casi exclusivamente centrada en los años santos y controlada por el régimen de Franco de inspiración Nacional-Católico (Pack, 2008), nace en París la Société Française des Amis de Saint Jacques de Compostelle. De su trabajo pionero destaca la recuperación de algunos caminos tradicionales jacobeos en Francia, su señalización, así como la creación de una primera credencial del peregrino en 1958. Este antecedente tiene continuidad en 1962 con la fundación en Estella (Navarra) de la primera asociación española de amigos del Camino cuyo objetivo era la difusión del Camino y la ayuda al peregrino. Posteriormente fueron apareciendo otras AAC, produciéndose el auténtico crecimiento a partir de la década de 1990.

Como se acaba de decir, la mayor parte de las AAC fueron fundadas entre la última década del siglo XX y la primera del XXI. Es decir, son posteriores al boom experimentado por el Camino a partir de 1993. Las más antiguas se encuentran en países y en localidades de larga tradición jacobea, no solo en España sino también en Francia, Alemania o Bélgica. Mientras, las de consitución más tardía aparecen en países en los que el fenómeno peregrinatorio a Compostela es más reciente, como América Latina, Asia o Europa del Este. Por número de asociados existen también importantes variaciones. En algunos casos se indica que al ser de carácter voluntario no existe la figura del asociado en si misma. Sin embargo, en la mayor parte de las encuestas se aporta una cifra que oscila entre $10 \mathrm{y}$ más de 2.000 miembros. Existe un reducido grupo, 11.2\%, con más de 1.000 asociados (la mayor tendría cerca de 3.000); y otro, aún menor, entre 500 y 1.000. Generalmente estas de mayor tamaño están en América del Norte (Estados Unidos y Canadá), Brasil y en algunos países europeos de tradición jacobea, como Francia, Bélgica o Alemania. Entre las más pequeñas están aquellas de países históricamente menos vinculados con Compostela, así como en España con una considerable fragmentación de las asociaciones.

En su devenir histórico es importante el año 1987 cuando se celebra el I Congreso Internacional de Asociaciones Jacobeas, creándose en el mismo acto una Coordinadora que será el germen para la constitución en 1993 de la Federación Española de Amigos del Camino de Santiago. En ambos casos, son fechas cargadas de simbolismo porque coinciden, en la primera, con la declaración del Consejo de Europa del Camino como Primer Itinerario Cultural Europeo y, en la segunda, con la nominación por parte de UNESCO de la ruta como bien Patrimonio de la Humanidad. En esos momentos iniciales, las AAC 
tuvieron un papel destacado en dos aspectos materiales: identificación y señalización del Camino; y creación y gestión de albergues para peregrinos. En estos primeros años, también organizan peregrinaciones en las que se trata de rememorar esa tradición, siendo frecuente portar los símbolos externos del peregrino compostelano.

El éxito contemporáneo del Camino, que se visibiliza en 1993 con casi 100.000 peregrinos, sólo se puede entender desde una perspectiva compleja en la que, casi siempre, es olvidado el papel de las AAC. Los dos polos, el político y el religioso, sobre los que se reconstruye la contemporaneidad del Camino no estarían completos si no se considera el rol de las AAC que ajenas, aunque muchas veces próximas, a aquéllos tuvieron un importante protagonismo en la puesta en valor del elemento más simbólico de la peregrinación: la ruta en si misma. Además, contribuyeron a mantener y difundir los valores del camino. De hecho, para Frey (1998) "The driving force behind their organization and formation was usually homecoming pilgrims who wishes to mantein a connection to the pilgrimage and to other pilgrims" (p. 207), constituyendo así lo que ella denomina una comunidad imaginada en la que la solidaridad es central (Lopez, 2013).

Esta misma inspiración era, básicamente, la que tenían en la Edad Media organizaciones similares a las AAC. A partir del siglo XII, por toda Europa (incluyendo los actuales territorios de Polonia o Islandia), fueron surgiendo cofradías, que se multiplican en los siglos posteriores, cuya misión era dar apoyo al peregrino (Rodríguez, 2018). Eran antiguos peregrinos que fundaban iglesias y albergues para dar hospitalidad a los caminantes y cualquier otra ayuda que precisaban. En algunos países su número era elevado, habiéndose identificado casi 40 en lo que hoy son los Países Bajos y Bélgica (Rodríguez, 2018), teniendo con frecuencia sus propios albergues, según ha constatado Herwaarden (Rodríguez, 2018 ). Rodríguez (2018) señala que la coincidencia en las funciones de las viejas y nuevas cofradías es tal que sólo parecen estar separadas por el tiempo. Por lo tanto, se podría decir que las necesidades que muestra un peregrino contemporáneo probablemente sean semejantes a las que tenía siete siglos atrás.

Los datos obtenidos del trabajo con las asociaciones, nos permiten afirmar que sus funciones actuales a penas han variado con el tiempo. El apoyo al peregrino es la más destacada. Incluye asesoramiento e información sobre todo lo relativo al itinerario. Como señala una de las personas entrevistadas, desdramatizar el camino. Hay que tener en cuenta que, con frecuencia, los peregrinos son personas mayores, que se tienen que enfrentar a importantes desafíos, como a un idioma que desconocen o atravesar territorios rurales con una despoblación aguda, todo ello durante muchas jornadas y a lo largo de varios cientos de kilómentros. Por lo tanto, ese apoyo se manifiesta en aportar información práctica y relevante. Pero no solo eso. Transmitir el significado de la peregrinación y la preparación mental, a veces también física, forma parte de su labor. En menor medida, destaca el apoyo al retorno, es decir acompañar el proceso de reincorporación a la vida diaria después de la peregrinación.

Esta labor inicial de asesoramiento e información que realizan de manera general todas las asociaciones, se complementa con otra, menos frecuente, que ofrece el $48.3 \%$ de las AAC, la preparación física y mental, como la organización de excursiones para el caso del entrenamiento físico, y charlas sobre el significado del Camino y de ser un peregrino o en el intercambio de experiencias, para la preparación mental. En relación a esta última, también hay un significativo porcentaje de AAC que dan apoyo al retorno, es 
decir al reingreso a la vida diaria de los peregrinos, a la incorporación de la experiencia peregrinatoria al quehacer cotidiano; o como señala una de las personas entrevistadas $U n$ packing the Way. La postexperiencia, que es un aspecto poco estudiado (Lopez, 2013), se convierte en un legado fundamental para las AAC porque sirve para nutrirlas de nuevos miembros al alargar en el tiempo los valores adquiridos en el viaje.

Además de las actividades señaladas anteriormente, hay otras muchas que son habituales. Por ejemplo, la entrega de credenciales, la señalización y mantenimiento de tramos del Camino, la organización de conferencias, cursos o la promoción de estudios jacobeos y la edición de guías. Algunas AAC también orientan sus esfuerzos hacia la investigación. Esta última característica fue muy importante no solo para evidenciar la relevancia histórica de las peregrinaciones, sino sobre todo porque permitió la recuperación de muchos itinerarios. De hecho, las AAC son en gran parte responsables de la incorporación de rutas y senderos que se habían perdido y por donde transitaban desde la Edad Media los peregrinos.

La gestión de albergues, propios o no, es marcada como una actividad por el $30.6 \%$. Una de las acciones más interesantes es la formación de voluntarios para trabajar en los albergues, ya sean de su propiedad o en otros de titularidad pública. Se trata de una actividad muy destacada que realizan algunas asociaciones, especialmente las más grandes, como puede ser la de Estados Unidos o la Confraternity of Saint James del Reino Unido, pero no de manera exclusiva. Algunas de estas asociaciones envían cada año hasta 70 u 80 hospitaleros voluntarios. La relevancia de esta iniciativa radica fundamentalmente en que estas personas no van a actuar como simples informadoras del Camino o como trabajadoras de un alojamiento. Su formación está basada en la tradición jacobea, en el significado del Camino y en la función de acogida de la peregrinación. Por lo tanto, la transmisión de la información y el trato al peregrino son acordes a los valores recibidos. Siguiendo a Wearing (2001), una de sus misiones sería la de evitar la comercialización y la banalización del Camino. Son agentes privilegiados para el mantenimiento del siginificado sobre el que se construyó esta vía de peregrinación. El altruismo, directamente relacionado con la ayuda y atención al peregrino es, junto con la hospitalidad, una pieza clave para entender el Camino como una vía de peregrinación.

Desde el punto de vista de la percepción actual y de las expectativas existentes, tanto las encuestas como las entrevistas nos dibujan un panorama, a veces, contradictorio. Preguntados sobre la situación general del Camino, la mitad de las encuestas indican que está bien, lo que unido a los que le dan la máxima puntuación, nos situamos en el 61.2\%. Solo algo menos del 10\% dicen que la situación es mala o muy mala. La opinión positiva bastante extendida, no quita que exista una visión crítica sobre ciertos aspectos de la ruta. Por ejemplo, más del $60 \%$ opina que el número de peregrinos es alto o demasiado alto durante la temporada alta. Con estas opiniones se refleja una idea que aparece de manera muy específica cuando se pregunta por el principal problema del Camino. En este caso, la masificación aparece como la respuesta más frecuente, siendo citada por el $43.5 \%$ de los encuestados.

Al igual que en las encuestas, las entrevistas también dejan entrever que la masificación es uno de los problemas centrales del Camino. De hecho, algunas de las personas más veteranas con las que hemos hablado nos han confesado que prefieren elegir caminos alternativos al Francés y, si es posible, en épocas del año con menos tránsito. En todo caso, las respuestas recibidas sobre este tema en las encuestas son coherentes con las 
manifestadas en la pregunta que interroga sobre las prioridades para mejorar la experiencia peregrinatoria. El 50\% de los encuestados optó por señalar "limitar la comercialización de la ruta”, uniendo así esta respuesta a la de la masificación. En la misma línea, sólo el $27,4 \%$ indica que la política promocional del Camino está bien o muy bien, reflejando la influencia que ha tenido sobre las dos cuestiones que se consideran principales: la masificación y la pérdida del sentido tradicional de la peregrinación.

Aunque no figuraba entre las preguntas del cuestionario, algunas observaciones escritas $\mathrm{y}$, sobre todo, las entrevistas han señalado un problema específico que afecta a las dos cuestiones citadas al final del párrafo anterior. Se trata de los 100 kilómetros mínimos que se requieren para obtener la Compostela. Los datos oficiales para 2019 señalan que algo más del $40 \%$ de los peregrinos comienzan la ruta en el límite de los $100 \mathrm{~km}$, convirtiendo a la localidad de Sarria (Lugo) en el principal punto de salida. De esta manera, una medida que se implementó para evitar que cualquier persona que llegase a Santiago pudiese demandar el certificado acreditativo, acabó por convertirse en una vía para la masificación y la pérdida del sentido tradicional de la peregrinación, según muchas de las personas entrevistadas. De hecho, la Fraternidad Internacional del Camino de Santiago elaboró un documento en 2016 titulado "Propuesta para elevar al ámbito de los $300 \mathrm{~km}$ la distancia mínima exigida para la entrega de la Compostela" (https://www.ficscaminodesantiago. com/). Dicha propuesta se basa tanto en criterios históricos como en otros prácticos con el fin de reducir la masificación.

Con todo, el tema de los $100 \mathrm{~km}$ evidencia la elasticidad de las AAC y el hecho de que a pesar de ser defensoras de la tradición muestran un carácter flexible. Aún reconociendo el problema adherido a los $100 \mathrm{~km}$ dejan ver que ese no es el único punto para focalizar las soluciones a la masificación. De hecho, justifican que pueda haber personas que por cuestiones de falta de tiempo o como aproximación inicial al mundo jacobeo, recorran esa distancia. Incluso en alguna ocasión afirman que caminar $100 \mathrm{~km}$ puede ser más meritorio que andar $800 \mathrm{~km}$.

La pérdida del sentido tradicional de la peregrinación, que es complementaria al predominio de la visión turística, es la segunda gran cuestión que surge del diálogo con las AAC, tanto en las encuestas como en las entrevistas. De nuevo aparece la cuestión de los $100 \mathrm{~km}$ como un tema central que, en general, favorece la proliferación de caminantes que buscan la obtención del certificado de una manera rápida sin que en verdad puedan apreciar el significado del Camino. Aunque el objetivo de esta investigación no es debatir sobre el tema de la motivación de los peregrinos, algunas respuestas, especialmente en las entrevistas, nos dejan ver elementos significativos.

El constante recurso a la idea del sentido tradicional de la peregrinación nos podría llevar a pensar en una defensa de los valores religiosos. Sin embargo, esa no es la opinión mayoritaria. Es cierto que, una entrevistada, nos dice que sólo entrega las credenciales en la iglesia, igual que defiende la necesidad de ir acompañada por explicaciones y ritos que le den significado a ese documento. También es verdad que de manera generalizada insisten en la necesidad de hacer trayectos largos para poder captar el espíritu del Camino. Pero todo esto no quiere decir que entiendan esta peregrinación desde una perspectiva religiosa, ni siquiera espiritual. En las encuestas, la motivación cultural/patrimonial fue señalada como la más habitual, por encima incluso de la espiritual; mientras, la religiosa 
fue considerada como la de menor relevancia entre los peregrinos actuales. Las entrevistas nos ayudan a enriquecer esta visión. No existe una ortodoxia a cerca de las motivaciones. Incluso en la amplia experiencia que tienen estas personas, indican que son variables en el espacio y en el tiempo: en ciertos momentos iniciales, antes del boom de 1993, hablan de una recreación de las peregrinaciones tradicionales, señalada también por Frey (1998). Ya en la actualidad se mezclan razones culturales, religiosas, espirituales e incluso deportivas que justifican su pasión por hacer el Camino. Aunque siempre, de fondo, está el concepto de valores tradicionales.

Para finalizar, un último grupo de preguntas hacía referencia al papel de las AAC. Los resultados son coherentes con otros obtenidos de las encuestas y entrevistas. La respuesta más común fue "mantener el sentido tradicional de la peregrinación", aunque también alcanzaron una representatividad importante la de "difusión de la cultura jacobea" y la "revitalización de la peregrinación". Esta cuestión era complementaria a otra que se interesaba por saber cual debería ser el papel de las AAC. De nuevo, encontramos dos respuestas muy mayoritarias: ayudar a mantener el sentido tradicional de la peregrinación y, sobre todo, difundir la cultura jacobea y dar apoyo al peregrino. Esta última fue citada por el $72.5 \%$ de los encuestados.

\section{ALTRUISMO, VOLUNTARIADO Y COLABORACIÓN}

Una vez vistos los principales resultados de las encuestas y entrevistas, se puede comenzar a reflexionar sobre su papel en el contexto de la gobernanza del Camino. Como ya hemos señalado, el protagonismo de las AAC ha sido minimizado en la investigación, pero también en la gestión de la ruta quedando como subsidiarias de las administraciones públicas y de la Iglesia. En este texto defendemos no sólo su papel central en la recuperación y difusión contemporánea del Camino, sino también su rol clave en el mantenimiento de la singularidad de los itinerarios jacobeos. Conceptos como altruismo o voluntariado son fundamentales para entender esta afirmación. Mientras, la colaboración con aquellas otras dos instituciones se considera muy importante para equilibrar los intereses en torno a los que se mueve el Camino..

En el nivel del gobierno formal del Camino aparecen dos instituciones principales. Por un lado, las administraciones públicas (en el caso español son principalmente las comunidades autónomas) que se encargan de la protección, promoción, planificación y gestión de las rutas. Por otro lado, la Iglesia no sólo en su papel simbólico como custodia del mito y de los ritos jacobeos, sino también como propietaria de buena parte del patrimonio a lo largo de la ruta y de muchos de los albergues de peregrinos. Además, es responsable de la emisión y homologación de la credencial oficial que es una certificación de paso que permite obtener la Compostela, registrada igualmente por la Iglesia.

A estas dos instituciones principales, organizadas jerárquicamente, hay que añadirle las AAC sin cuya existencia es imposible entender el Camino de Santiago. Se trata, como se dijo, de asociaciones de carácter voluntario muy diversas y con capacidad de acción desigual. Su labor se puede englobar bajo el paraguas del altruismo. Paraskevaidis y Andriotis (2017) han analizado el complejo y controvertido concepto de altruismo en el ámbito del turismo. Estos autores debaten sobre altruismo recíproco y verdadero, iden- 
tificando este último como aquel "without expected favours in return" (p. 28). Señalan que el auténtico altruismo encajaría en la Social Exchange Theory a través de lo que Blau (1964) denomina recompensas de carácter intríseco, como el reconocimiento y el respeto, por ejemplo. Con todo, para Paraskevaidis y Andriotis (2017) es mucho más apropiado utilizar la teoría del Altruistic Surplus Phenomenon-ASP al estar conectada con la idea de beneficios colectivos a la comunidad estando, pues, más vinculada con el concepto de alturismo recíproco (Paraskevaidis y Andriotis, 2017). En todo caso, el altruismo, entendido como ayuda al otro (verdadero o recíproco), forma parte de la tradición de muchas religiones y se manifiesta también en lo que Einholf (2011) denomina prosocial behavior, conectando así el Camino con sus orígenes religiosos y con otro de sus elementos fundamentales, la hospitalidad. En este mismo ámbito del altruismo, Buzinde, Kalavar, Kohli y Manuel-Navarrete (2014) en su investigación sobre una peregrinación hinduista, se refieren a dos tipos de actividades realizadas por los peregrinos: activities Serving self y activities Serving others. Estas últimas involucran sobre todo a peregrinos locales y permiten satisfacer las denominadas activities serving self. Estas actividades se vincularían con dos tipos de experiencias, las espirituales y las de solidaridad social (Buzinde et al., 2014) que se relacionaría con el concepto de existential communitas de Turner (1969).

En relación al voluntariado altruista se encuentra el fenómeno de la hospitalidad. Personas normalmente formadas por las AAC, por aquellas con más capacidad, que durante un tiempo determinado trabajan de manera desinteresada en albergues y puntos de información dando apoyo al peregrino y contribuyendo a transmitir el significado tradicional de la ruta. Estas personas son técnicamente turistas, volunturistas (Hernandez-Maskikver, Lapointe y Aquino, 2018), si bien no responden a ninguna de las tipologías que indican autores como Wright (2013), puesto que se rompe esa visión binaria entre turista y huesped. De hecho, serían turistas actuando como huéspedes. Wright (2013) señala las dificultades para definir al turismo voluntario aunque indica los beneficios educacionales que genera y, lo más importante, que ese carácter altruista debe de ser matizado porque hay un enriquecimiento mútuo entre quienes interactúan de este modo.

En sus reflexiones sobre el turismo como una catálisis para una autenticidad existencial, Brown (2013) pone especial énfasis, entre otros, en el turismo de voluntarios, destacando aquellos estudios que evidencian esas transformaciones existenciales que se producen y que rompen con la autenticidad de la vida diaria. Por su parte, Morpeth (2012) considera el papel crucial de los voluntarios en la acogida a los visitantes de lugares religiosos menores. Aunque la definición tradicional (Wearing, 2001) relaciona el voluntariado con beneficios para las comunidades locales, otras lecturas más críticas han enfatizado también la existencia de impactos negativos (Guttentag, 209; Hernandez-Maskikver et al., 2018) o la comercialización de su labor (Coren y Gray, 2012; Wearing, 2001). De cualquier manera, a diferencia de otros casos, el voluntariado a lo largo del Camino está más centrado en interactuar con los peregrinos que con las comunidades locales.

Volviendo a las asociaciones, el altruismo demostrado hacia la peregrinación tiene su recompensa, que se puede manifestar de formas diferentes. De no producirse, podría derivar en una menor motivación hacia su labor (Wright, 2013). O'Regan y Choe (2019) analizan la transformación del Couchsourfing-CS hacia una fórmula comercial y las consiguientes tensiones que se generaron con su modelo de voluntariado. Estos dos autores 
se apoyan en la Sacred Value Protection Model, vinculada con la Economía Moral. Al igual que entre los embajadores de CS existían unos valores compartidos basados en el altruismo, la generosidad o la abnegación, entre otros, en el caso de las AAC encontramos unas actitudes semejantes de compromiso hacia el Camino y sus valores, la comunidad imaginada de Frey (1998). Por eso puede resultar de especial interés considerar, al igual que pasó con el CS, las consecuencias de un modelo de gestión del Camino que no tenga en consideración los valores y aspiraciones de las AAC. Si seguimos con el ejemplo de $\mathrm{CS}$, las implicaciones podrían derivar en desconfianza y menor compromiso con el poder formal que representan la Iglesia y las administraciones públicas.

En general, existe una colaboración estrecha entre estas dos instituciones que representan el gobierno formal del Camino sin que haya conflictos aparentes. Se trata de una colaboración beneficiosa entre quien detenta las competencias públicas y quien posee el poder simbólico. Citando a Bourdieu (1991), Terzidou et al. (p. 56, 2018) dicen que "Religious institutions structure the dependence of people on religious symbolic power". Además, esta colaboración en el ámbito del turismo refuerza unas relaciones que hay que entenderlas en su dimensión temporal (Lee, Riley y Hampton, 2009) y que para el caso del Camino tienen una larga tradición. Otros actores implicados, como los municipios o las asociaciones de amigos quedan relegados a un plano secundario. En este último caso, estamos delante de organizaciones con un carácter de voluntariado y laico que son independientes tanto de las administraciones públicas como de la Iglesia. Esta independencia no quita para que no compartan intereses comunes si bien con objetivos finales diferentes: económicos (gobiernos), religiosos (Iglesia) y mantenimiento de la tradición (AAC). Los muchos puntos en común permitirían un trabajo en red.

Powell (1990) se refiere a la importancia de la organización en redes frente, por ejemplo, a los modelos jerárquicos. Establece tres condiciones que, a su entender, son componentes cruciales de esta forma de organización: Know-how; rapidez, es decir la capacidad para difundir e interpretar de forma rápida la información, para lo que se precisan canales eficientes de comunicación; y confianza. Se trata, en definitiva, de una forma de colaboración que puede resultar de gran valor para conciliar los intereses de las distintas instituciones que actúan en el Camino de Santiago por cuanto se dan las condiciones señaladas por Powell (1990).

En el ámbito de las teorías de la colaboración, de las 5 propuestas que hacen Fyall, Garrod y Wang (2012), hay dos que nos interesan especialmente: las politics-based theories, que tienen que ver con las relaciones de poder; y, sobre todo, las relationship-based theories, conectadas con la dependencia mutua y con la creación de redes. En relación a esta última teoría, la colaboración no implica que cada parte deje de trabajar en aquello que le genera más interés. Abordar un problema común constituye una forma de estimular esa cooperación, siendo esta más atractiva en la medida en la que los problemas son más complejos. En todo caso, se necesita confianza y compromiso, así como desdibujar las relaciones de poder que pudiesen existir (Fyall et al., 2012).

Farsari (2018) recurre a las teorías de la representaciones sociales de Moscovici (2001) para entender los procesos de colaboración, bajo la idea de que las creencias compartidas socialmente permiten una mejor comunicación e interacción. De nuevo Farsari (2018), siguiendo a Moscovici (1988), señala que hay tres formas en las que una representation se 
convierte en social: la hegemónica, la emancipada y la polémica. La segunda de ellas no entra necesariamente en conflicto con la primera sino que la complementa. En definitiva y recurriendo al trabajo clásico de Gray (1989), la colaboración puede servir para avanzar cuando existen visiones compartidas, y para resolver conflictos.

En relación a este último término, Curcija, Breakey y Driml (2019) realizan una exhaustiva revisión de la literatura sobre el conflicto, especialmente en la gestión y entre los stakeholders, con atención al turismo de base comunitaria. Una de las ideas que recogen es que el conflicto no siempre es destructivo, que puede ser una oportunidad para el cambio. Señalan que es necesario identificar el conflicto para evitar exacerbar problemas futuros, así como entenderlo. Incorporan también la idea de las soluciones creativas. McKercher, Ho y du Cros (2005) indican que el conflicto es más probable que se produzca en situaciones de desequilibrio de poder y en escenarios dinámicos, cambiantes. Mientras, Deutsch (2006) dice que la existencia de conflicto lleva implícita alguna forma de interdependencia y de falta de comunicación (Almeida, Costa y Silva, 2017).

\section{LAS ASOCIACIONES DE AMIGOS DEL CAMINO Y SUS RELACIONES CON OTRAS INSTITUCIONES}

Los resultados obtenidos de las encuestas y entrevistas nos han permitdo obtener una visión general del papel que tienen las AAC en la peregrinación jacobea actual. Frente a los intereses primarios de las administraciones públicas y de la Iglesia, las AAC focalizan sus esfuerzos en la conservación del sentido y de los valores tradicionales de la peregrinación a Santiago. El significado que tienen estos últimos conceptos no son fáciles de explicar pero se relacionarían con ideas como la hospitalidad no comercial, el sentido de comunitas o la oposición a la mercantilización y a la simplificación del Camino. Esta idea contra la simplificación puede resultar en si misma contradictoria porque el discurso dominante es bastante flexible y aunque esto es una buena muestra de la complejidad de las AAC, al mismo tiempo refleja una aproximación que relativiza y justifica muchas actitudes de tal forma que parece simplificarlas.

En todo caso, la simplicidad que rechazan las AAC tiene que ver, básicamente, con la conversión del Camino en un producto turístico y todo lo que ello lleva implícito. Sin embargo, lejos de cualquier ortodoxia dan prueba, aquí también, de un relativismo que podría llegar a confundirse con simplificación. Algunos ejemplos nos ayudan a entender esta idea. En primer lugar la motivación. Los resultados de las encuestas señalan que las principales motivaciones actuales serían las de tipo cultural-patrimonial, espiritual y natural, ocupando el último puesto la de carácter religioso. Pero es en las entrevistas en donde mejor se aprecia la adaptabilidad del discurso. Reconocen que hay múltiples razones para hacer el Camino y que no son excluyentes: espiritual, cultural, deportiva... Advierten, además, que la popularidad adquirida a través de libros o películas de gran proyección pública, ha ayudado a reforzar el papel transcendental de la ruta ya que acostumbran a transmitir una imagen muy idealizada y espiritual de la ruta.

En segundo lugar, aún reconociendo los prejuicios de la masificación, citada como el primer problema, la postura de las AAC sigue siendo muy flexible. Ya vimos anteriormente esa relativización de los $100 \mathrm{~km}$ que es donde se producen, especialmente en el verano, las 
principales aglomeraciones. Pero incluso con la masificación hay una cierta elasticidad que lleva a que no se posicionen en el debate sobre verdaderos/falsos peregrinos. Uno de los entrevistados señala que no se puede decir que haya buenos o malos peregrinos, que eso está en la conciencia de cada uno. Más explícito es otra de las personas con las que hablamos cuando dice que hay que tratar a los turistas con comprensión porque así pueden convertirse en peregrinos. Por lo tanto, aún a pesar de que denuncian la masificación, también encuentran oportunidades en la misma, tanto en el sentido de communitas o encuentro con el otro, como en el sentido más introspectivo, de encuentro con uno mismo.

La flexibilidad que exhiben las AAC en su comprensión del mundo jacobeo, no evita la crítica, también desde posiciones pragmáticas. Por ejemplo, entienden la misión de las administraciones públicas en relación al fomento del crecimiento económico a pesar de que genere comercialización de la ruta. Igualmente, reconocen la labor de la Iglesia, aunque hay un sentimiento de abandono por su parte. Es cierto, e insisten en ello, que las AAC son de carácter laico por lo que su perfil no puede ser religioso. Sin embargo, se espera que haya valores compartidos como el sentido de la hospitalidad o el de comunidad, que ayuden a intensificar su colaboración. Estos argumentos de necesaria cooperación con la Iglesia y, al mismo tiempo, de cierto recelo hacia ella aparecen perfectamente recogidos por John Raferty (2013) de la Confraternity of Saint James. En el texto presentado durante la celebración del I Congreso Internacional de Acogida Cristiana y Nueva Evangelización, organizado por el Cabildo de la Catedral de Santiago en 2013, habla de las vías de convergencia entre Iglesia y AAC, poniendo la acogida en el centro de su discurso; y aún destacando el carácter seglar de las AAC defiende la colaboración entre las dos instituciones aunque sin disimular una cierta decepción cuando dice que "Sería fácil para nosotros ser críticos con la Iglesia y otros grupos religiosos" (p. 71). Frey (1998) también recoge en su obra algunas de esas críticas de los peregrinos a la Iglesia.

Sin embargo, resulta difícil hablar de conflicto entre las partes, no sólo porque las AAC buscan preservar su independencia y fortalecer la concordia, sino también porque el mensaje de la Iglesia y de las administraciones públicas conserva mucho contenido histórico y cultural. De hecho, el discurso hegemónico, tanto de la Iglesia como del gobierno, sigue siendo que el Camino es mucho más que un producto turístico, es un itinerario cultural con un contenido religioso y espiritual evidente. Además, la escasa relevancia social y la falta de unanimidad por parte de las asociaciones, hace que no se pueda hablar de aquella tercera forma de representación, la polémica, que Farsari (2018) tomaba de Moscovici (1998).

Las administraciones públicas, sobre todo el Gobierno de Galicia, se han beneficiado del reconocimiento internacional de una ruta que ha orientado buena parte de su política turística y que ha generado un importante número de visitantes. Mientras, la Iglesia como institución y particularmente la Compostelana, ha reforzado sus lazos con el Estado, a pesar de la separación oficial que marca la Constitución española. Un ejemplo lo representan las visitas papales a Santiago en cuyo coste y organización participan activamente las administraciones públicas. También se puede citar la propia comercialización de souvenires (la catedral dispone de 2 tiendas), o de servicios turísticos, como el museo, las cubiertas o el pórtico de la Gloria en el templo compostelano.

Como hemos dicho, la representación hegemónica del Camino, sustentada en un desequilibrio de poder puede facilitar la transición de las asociaciones hacia una representa- 
ción polémica en la medida en que esas desigualdades alimentan la desconfianza (Cook, Hardin y Levi, 2005). En ese conflicto, escasamente visible, resulta difícil desafiar la representación hegemónica por cuanto el discurso público dominante, que no la acción, continua estando muy próximo al de la representación emancipada. En todo caso, la falta de armonía entre discurso y acción puede llevar hacia una desconfianza, y consiguiente exclusión, de aquellos grupos, las asociaciones, más críticos con el devenir del Camino. En su lectura foucauliana del poder Nankoo y Ramkissoon (2011) señalan que una situación de desconfianza, como esta, podría generar parálisis y un sentimiento de desempoderamiento por parte de aquellos grupos que ya se perciben a si mismos como menos poderosos. Inevitablemente esto conduciría a una pérdida de confianza con todo lo que ello significa en términos de colaboración (Nankoo y Ramkissoon, 2011).

Los resultados de las encuestas nos indican que hay un sentimiento dominante de que las AAC no son tenidas en cuenta por esos dos actores principales (59\%), de forma particular varias respuestas hacer referencia específica al abandono por parte de la Iglesia. Tenemos que recurrir a las desiguales relaciones de poder para entender su exclusión del proceso colaborativo (Lee et al., 2009); de hecho, Buzinde et al. (2014) afirman que la cooperación es más probable cuando todos los miembros de un grupo se sienten incluidos. El sentimiento de exclusión se agrava por una cierta perspectiva crítica que caracteriza a algunas asociaciones cuando reflexionan sobre la situación actual del Camino. La confianza, como una de las bases para la organización en red y la colaboración (Fyall et al., 2012; Powell, 1990) resulta fundamental, igual que lo es redirigir las relaciones de poder hacia una estructura más horizontal. Problemas complejos, como la masificación de ciertos tramos de la ruta, podrían ser abordados de manera mucho más eficaz desde esta perspectiva.

En el caso del Camino encontramos una situación paradójica en las relaciones entre los tres actores principales. En el triángulo que se establece entre (a) asociaciones, (b) Iglesia y (c) gobierno, las creencias compartidas entre a y b son mucho más próximas, a pesar de que la colaboración se produce fundamentalmente entre b y c. De hecho, uno de los entrevistados llega a decir que los caminos entre la Iglesia y las AAC en estos momentos son paralelos, sin ningún punto de convergencia. Significa esto que las relaciones de poder son más importantes que aquellas otras derivadas de unas creencias socialmente compartidas de las que habla Farsari (2018) cuando intenta entender los procesos de colaboración. El mismo entrevistado justifica esa falta de colaboración de la Iglesia por un problema generacional en el que pesa demasiado el envejecimiento del clero.

Una de las cuestiones recurrentes en el trabajo de campo es el deseo de las AAC por mantener su autonomía de acción. Esto no quiere decir que no deseen colaborar con otras instituciones implicadas. Su labor encaja en lo que Paraskevaidis y Andriotis (2017) denominan altruimo verdadero puesto que no buscan ninguna contrapartida, aunque sí reclaman reconocimiento y respeto, eso que Blau (1964) llamaba recompensas de carácter intrínseco. Con todo, podemos considerar que la labor de las AAC encajaría también en la ASP de Paraskevaidis y Andriotis (2017) en la medida en la que su labor genera beneficios colectivos al Camino al contribuir a su difusión y a la transmisión de una tradición de peregrinación que va mucho más allá de la meramente turística, contribuyendo así a su éxito internacional. O también a la idea desarrollada por Buzinde et al. (2014) 
cuando afirman que las actividades asociadas con servir al otro responden a una actitud de altruismo ligada a una búsqueda más profunda de uno mismo.

\section{CONCLUSIONES}

Las AAC son fundamentales para entender la recuperación contemporánea del Camino de Santiago, su éxito internacional y su carácter complejo que va mucho más allá de un simple producto turístico. Su trabajo es muy poco conocido y reconocido, asociándolas, con frecuencia, y de manera errónea, con un carácter religioso. Si bien en su composición muestran una gran heterogeneidad uno de los elementos que le dan cohesión es su preocupación por la conservación de los valores tradicionales del Camino que se centran principalmente en la hospitalidad y en el sentido de communitas que se desarrolla a lo largo del proceso peregrinatorio, que no es solo físico sino también mental.

En este contexto de conservación y reconocimiento de los valores tradicionales de la peregrinación, más allá de los de tipo religioso, las AAC se encuentran en una posición de debilidad institucional frente a las administraciones públicas, más interesadas en aspectos puramente turísticos, y a la propia Iglesia, centrada en preocupaciones pastorales pero también en otras más materiales y próximas a las de las administraciones. Todo esto a pesar de que el discurso hegemónico continua siendo el de un itinerario histórico que trasciende el concepto puramente turístico.

Las realidades divergentes que se dibujan son el de una multiplicación en el número de caminantes que provoca problemas de masificación en ciertos momentos del año y en algunos tramos finales del Camino Francés; y el de una percepción por parte de las AAC de que la ruta jacobea es cada vez más un producto turístico. De hecho, un tercio de las AAC opinan que en la actualidad el Camino es sobre todo eso, un producto turístico. Sin embargo, la creencia expresada por los otros dos tercios de que continúa siendo una vía de peregrinación evidencia que, aún a pesar de todo, hay suficientes elementos para seguir creyendo en el Camino como una ruta histórica de peregrinación que conserva, al menos en parte, sus valores tradicionales.

Esta resistencia que muestran las AAC evita la existencia de un conflicto con las instituciones del gobierno formal, en la medida en la que consideran que su labor en relación al Camino permite mantener esos valores tradicionales. Sin embargo, tanto las encuestas como las entrevistas dejan entrever algunas debilidades que pueden condicionar el futuro de las AAC y, en consecuencia, del propio Camino. Nos centraremos en dos que están interconectadas: la pérdida de algunas de sus funciones y la falta de relevo generacional. Con respecto a la primera, la investigación y el estudio de las peregrinaciones históricas llevó a la recuperación de antiguos trazados, su señalización y mantenimiento. Pasados más de 50 años, esa fase, que generó un gran entusiasmo, fue superada quedando en muchos casos reducida a labores de mantenimiento. Ese momento de descubrimiento acercó al fenómeno jacobeo a gente joven con iniciativa; de hecho, algunos de los pioneros en el movimiento asociativo jacobeo provienen de las universidades, como es el caso de la Asociación Americana u otras en Europa.

La falta de relevo generacional es otro problema al que se enfrentan las AAC y que condiciona tanto su futuro como su dinámica actual. En esta investigación no se ha pro- 
fundizado en sus causas, que pueden ser comunes al movimiento asociativo en general, pero que también puede obedecer a razones propias, como esa pérdida de funciones a la que hemos hecho alusión. Es cierto que se mantienen muchas de sus actividades, pero no la que marca su carácter pionero. Tal vez, la más relevante en estos momentos sea la de la capacitación de voluntarios que actuarán como embajadores de los valores del Camino; también la difusión a través de las redes sociales. Sin embargo, no todas las AAC tienen capacidad para realizar estas tareas.

Estas debilidades de las AAC las hacen más vulnerables y sensibles a la marginación y, por lo tanto, al conflicto y a la desmotivación. Como señalan Cook, Hardin y Levi (2005), el desequilibrio de poder, en este caso en aumento, genera más desconfianza y desigualdad, creando un ambiente propicio para el conflicto. Con todo, como hemos visto, al menos hasta el momento, no se observa una tendencia hacia el mismo y el proceso de envejecimiento de las AAC tampoco lo facilita. Sin embargo, la desmotivación de la que nos hablaba Wright (2013) podría ser una vía mucho más eficaz para acelerar el debilitamiento y marginación da las AAC. En un acto de autocrítica, una de las personas entrevistadas señala que en España, junto al envejecimiento, otro problema de las asociaciones es un cierto carácter endogámico y personalista que no facilita la incorporación de nuevos miembros.

En un escenario de desmotivación ¿Que pasaría si las AAC desaparecen del Camino o si su papel se reduce sustancialmente? Las implicaciones podrían ser varias. En primer lugar, de carácter material. Las AAC llevan adelante acciones de conservación de las rutas, de señalización o de gestión de albergues públicos y privados. Estas tareas tendrían que ser asumidas por las administraciones, sustituyendo muchas veces la labor historico-cultural de las AAC por la profesional de carácter turístico de un empleado. Las AAC son fundamentales para entender la singularidad del Camino como ruta de peregrinación más allá de los elementos religiosos, como puede ser la hospitalidad, el voluntariado o esa dimensión histórico-cultural. Además, tienen una función muy activa en la internacionalización. De acuerdo con la opinión de alguna de las personas entrevistadas, sin las AAC el Camino acabaría convirtiéndose en un producto turístico más. Aún reconociendo la diversidad del concepto de peregrinación y esa frontera líquida con el turismo, y considerando el turismo como parte esencial de la peregrinación y que "tourist moments and activities are considered an essencial part of a religious journey" (Terzidou et al., 2017, p. 126), creemos que el concepto de peregrinación no es intercambiable con el de turismo y debe mantener algunos elementos diferenciadores relacionados con sus valores espirituales, aunque no siempre religiosos como, por el contrario, señala Terzidou et al. (2017).

Por lo tanto, parece evidente la necesidad de empoderar a las AAC como estrategia para conservar la singularidad como ruta de peregrinación que trasciende lo puramente religioso o turístico. Incluso se puede ir más allá acudiendo a Frey (1998) cuando relaciona el éxito de las AAC con la postexperiencia, labor fundamental de las asociaciones, y la capacidad para favorecer un cambio social en el que emociones adquiridas durante la peregrinación, como solidaridad, humildad o felicidad (Lopez, 2013) son importantes en el regreso a casa. Si bien podemos concluir que no hay conflicto abierto entre las AAC y las administraciones públicas y la Iglesia, las desigualdades de poder existentes son un argumento para el conflicto (McKercher et al., 2005) o la desmotivación. 
Siguiendo a autores como Almeida et al. (2017) o Curcija et al. (2019), prevenir el conflicto implica reconocerlo, identificarlo, aunque sea en su forma potencial, y analizarlo. La idea que recogen Curcija et al. (2019) de que el conflicto puede ser una oportunidad para el cambio resulta estimulante, sobre todo si consideramos que no estamos hablando de incompatibilidad entre los stakeholders, ni siquiera de choque da valores (McKercher et al., 2005). Al contrario, es fruto de una interdependencia (Deutsch, 2006) en la que reconocer las ventajas del trabajo conjunto, la colaboración mútua, permite resolver conflictos (Gray, 1989).

Declaración responsable: $\mathrm{El}$ autor declara que no existe ningún conflicto de interés relacionado con la publicación de este artículo.

\section{BIBLIOGRAFÍA}

ALMEIDA, J., COSTA, C. y NUNES DA SILVA, F. (2017): «A framework for conflict analysis in spatial planning for tourism», Tourism Management Perspectives, vol. 24, pp. 94-106.

BLAU, P. (1964): Exchange and power in social life. Nueva York, Wiley.

BLOM, T., NILSSON, M. y SANTOS, X. (2016): «The Way to Santiago beyond Santiago. Fisterra and the pilgrimage's post-secular meaning», European Journal of Tourism Research, vol. 12, pp. 133-146.

BOURDIEU, P. (1991): «Genesis and structure of the religious field», Comparative Social Research, vol. 13, pp. 1-44.

BROWN, L. (2013): «Tourism: A catalyst for existential authenticity», Annals of Tourism Research, vol. 40, pp. 176-190.

BUZINDE, C.N., KALAVAR, J.M., KOHLI, N. y MANUEL-NAVARRETE, D. (2014): «Emic understandings of Kumbh Mela pilgrimage experiences», Annals of Tourism Research, vol. 49, pp. 1-18.

COLLINS-KREINER, N. (2010): «Researching the pilgrimage: Continuity and transformations», Annals of Tourism Research, vol. 37 (2), pp. 440-456.

COLLINS-KREINER, N. (2018): «Pilgrimage-tourism: common themes in different religions», International Journal of Religious Tourism and Pilgrimage, vol. 6 (1), pp. 8-17.

COOK, K.S., HARDIN, R. y LEVI, M. (2005): Cooperation without trust? Nueva York, Russell Sage Foundation.

COREN, N. y GRAY, T. (2012): «Commodification of volunteer tourism: A comparative study of volunteer tourists in Vietnam and in Thailand», International Journal of Tourism Research, vol. 14 (3), pp. 222-234.

CURCIJA, M., BREAKEY, N. y DRIML, S. (2019): «Development of a conflict management model as a tool for improved project outcomes in community based tourism», Tourism Management, vol. 70, pp. 341-354.

DEUTSCH, M. (2006): «Cooperation and Competition», en DEUTSCH, M., P.T. COLEMAN, P.T. y MARCUS, E.C. (Eds.): The handbook of conflict resolution. Theory and practice. San Francisco, Jossey-Bass, PP. 23-42. 
DUNN, M. (2016): «Historical and modern signs of "real" pilgrims on the road to Santiago de Compostela», en SANCHEZ y SANCHEZ, S. y HESP, A. (Eds.): The Camino de Santiago in the 21st century. Nueva York, Routledge, pp. 13-35.

EINHOLF, C.J. (2011): «The Link Between Religion and Helping Others: The Role of Values, Ideas and Language», Sociology of Religion, vol 72 (4), pp. 435-455.

FARSARI, I. (2018): «A structural approach to social representations of destination collaboration in Idre, Sweden», Annals of Tourism Research, vol. 71, pp. 1-12.

FREY, N. (1998): Pilgrim stories: On and off the road to Santiago. Berkeley, University of California Press.

FYALL, A., GARROD, B. y WANG, Y. (2012): «Destination collaboration: A critical review of theoretical approaches to a multi-dimensional phenomenon», Journal of Destination Marketing \& Management, vol. 1 (1-2), pp. 10-26.

GRAHAM, B. y MURRAY, M. (1997): «The spiritual and the profane: the pilgrimage to Santiago de Compostela», Ecumene, vol. 4 (4), pp. 389-409.

GRAY, B. (1989): Collaborating: Finding Common Ground for Multiparty Problems. San Francisco, Jossey-Bass.

GUTTENTAG, D.A. (2009): «The possible negative impacts of volunteer tourism», International Jounal of Tourism Research, vol. 11 (6), pp. 537-551.

HERNANDEZ-MASKIVKER, G., LAPOINTE, D. y AQUINO, R. (2018): «The impact of volunteer tourism on local communities: A managerial perspective»,International Journal of Tourism Research, vol. 20 (5), pp. 650-659.

HOWARD, C. (2012): «Speeding up and slowing down: Pilgrimage and slow travel through time», en Fullagar, S., Markwell, K. y Wilson, E. (Eds.) Slow tourism: Experiences and mobilities. Bristol, Channel View Publications, pp. 11-24.

KIM, B., KIM, S.S. y KING, B. (2016): «The sacred and the profane: Identifying pilgrim traveler value orientations using means-end theory», Tourism Management, vol. 56, pp. 142-155.

LEE, T.J., RILEY, M. y HAMPTON, M.P. (2010): «Conflict and Progress. Tourism Development in Korea», Annals of Tourism Research, vol. 37 (2), pp. 355-376.

LOIS-GONZÁLEZ, R.C. y SANTOS, X.M. (2015): «Tourists and pilgrims on their way to Santiago. Motives, Camino sand final destinations», Journal of Tourism and Cultural Change, vol. 13 (2), pp. 149-164.

LOPEZ, L. (2013): «How long does the pilgrimage tourism experience to Santiago de Compostela last?», International Journal of Religious Tourism and Pilgrimage, vol. 1 (1), pp. 1-14.

MACLEAN, K (2009): «Seeing, being seen, and not being seen: Pilgrimage, tourism and layers of looking at the Kumbh Mela», Cross Currents, vol. 59 (3), pp. 319-341.

MAGRY, P.J. (2015): «To be or not to be ... a pilgrim. Spiritual pluralism along the Camino Finisterre and the urge for the end», en Sánchez-Carretero, C. (Ed.): Heritage, Pilgrimage and the Camino to Finisterre. Heidelberg, Springer, pp. 175-211.

McKERCHER, B., HO, P.S.Y. y DU CROS, H. (2005): «Relationship between tourism and cultural heritage management: evidence from Hong Kong», Tourism Management, vol. 26 (4), pp. 539-548. 
MORPETH, N. (2012): «The role of volunteers in the provision of faith tourism in the North of UK», International Journal of Tourism Policy, vol. 4 (2), pp. 106-116.

MOSCOVICI, S. (1988): «Notes towards a description of social representations», European Journal of Social Psychology, vol. 18 (3), pp. 211-250.

MOSCOVICI, S. (2001): "Why a theory of social representations», en DEAUX, K. y PHILOGENE, G. (Eds.): Representations of the social: Bridging theoretical traditions. Oxford, Blackwell, pp. 8-35.

MURRAY, M. y GRAHAM, B. (1997): «Exploring the dialects of route-based tourism: the Camino de Santiago», Tourism Management, vol. 18 (8), pp. 513-524.

NANKOO, R., y RAMKISSOON, H. (2011): «Power, trust, social exchange and community support», Annals of Tourism Research, vol. 39 (2), pp. 997-1.023.

NILSSON, M. y TESFAHUNEY, M. (2016): «Performing the "post-secular" in Santiago de Compostela», Annals of Tourism Research, vol. 57, pp. 18-30.

PRITCHARD, A., MORGAN, N. y ATELJEVIC, I. (2011): «Hopeful Tourism: A New Transformative Perspective», Annals of Tourism Research, vol. 38, pp. 941-963.

O'REGAN, M. y CHOE, J. (2019): «Managing a non-profit hospitality platform conversion: The case of Couchsurfing.com», Tourism Management Perspectives, vol. 30, pp. 138-146.

OLSEN, D.H. y WILKINSON, G. (2016): «Are fast pilgrims true pilgrims? The Shikoku pilgrimage», Annals of Tourism Research, vol. 61, pp. 228-230.

PARASKEVAIDIS, P. y ANDRIOTIS, K. (2017): «Altruism in tourism: Social Exchange Theory vs Altruistic Surplus Phenomenon in host volunteering»,Annals of Tourism Research, vol. 62, pp. 26-37.

PACK, S. (2008): «The Camino de Santiago and the paradox of national Catholicism in modern Spain», en Bunk, B., Pack, S.y Scott, C.G. (Eds.) Nation and Conflict in Modern Spain: Essays in honor of Stanley G. Payne. Madison, University of Madison Press pp. 65-88.

PACK, S. (2010): «Revival of the pilgrimage to Santiago de Compostela: The politics of religious, national, and European patrimony, 1879-1988», The Journal of Modern History, vol. 82 (2), pp. 335-367.

POWELL, W.W. (1990): «Neither market nor hierarchy: Network forms of organization», Research in Organizational Behavior, vol. 12, pp. 295-336.

RAFFERTY, J. (2013): «Acogida a peregrinos creyentes y no creyentes», en Actas del I Congreso Internacional de Acogida Cristiana y Nueva Evangelización. Santiago, Catedral de Santiago, pp. 63-71. Disponible en http://acogidacristianaenloscaminosdesantiago.org/wp-content/uploads/2018/02/I-Congreso-de-Acogida-Cristiana.pdf

RODRÍGUEZ, M.F. (2018): «Función de las asociaciones de amigos del Camino. Sus conexiones con las antiguas cofradías de peregrinos a Santiago», en Actas del VI Congreso Internacional de Acogida Cristina en el Camino de Santiago. Santiago, Catedral de Santiago, pp. 163-173. Disponible en http://archicofradia.org/wp-content/ uploads/2019/07/6-d-MANUEL-F.-RODRIGUEZ-2.pdfhttp://archicofradia.org/wpcontent/uploads/2019/07/6-d-MANUEL-F.-RODRIGUEZ-2.pdf

SANCHEZ y SANCHEZ, S. y HESP, A. (Eds.) (2016): The Camino de Santiago in the 21 st century. Interdisciplinary perspectives and global views. Nueva York, Routledge. 
SANTOS, X.M. (2016): «The Way of Saint James as an event: Politics and nation», Journal of Policy Research in Tourism, Leisure and Events, vol. 8 (8), pp. 233-248.

SANTOS, X.M. (2017): «La construction touristique des chemins de Saint-Jacques-deCompostelle en Espagne: acteurs, regards et contradictions», Sud-Ouest Européen, vol. 43, pp. 7-19.

SANTOS, X.M. y TRILLO-SANTAMARÍA, J.M. (2017): «Tourism and nation in Galicia (Spain) », Tourism Management Perspectives, vol. 22, pp. 98-108.

SECOR, A.J. (2010): «Social Surveys, Interviews and Focus Groups», en GOMEZ, B. y JONES III, J.P. (Eds.): Research Methods in Geography. A Critical Introduction. Chichester, John Wiley \& Sons, pp. 194-205.

TERZIDOU, M., SCARLES, C. y SAUNDERS, M.N.K. (2017): «Religiousness as tourist performances: A case study of Greek Orthodox pilgrimage», Annals of Tourist Research, vol. 66, pp. 116-129.

TERZIDOU, M., SCARLES, C. y SAUNDERS, M.N.K. (2018): «The complexities of religious tourism motivations: Sacred places, vows and visions», Annals of Tourism Research, vol. 70, pp. 54-65.

TIMOTHY, D. y OLSEN, D. (2006): Tourism, Religion and Spiritual Journeys. Londres, Routledge.

TURNER, V. (1969): The ritual process: Structure and anti-structure. Chicago, Aldine publ.

WEARING, S. (2001): Volunteer Tourism: Experiences that Make a Difference. Nueva York, CABI Publishing.

WRIGHT, H. (2013): «Volunteer tourism and its (mis)perceptions: A comparative analysis of tourist/host perceptions», Tourism and Hospitality Research, vol. 13 (4), pp. 239250. 